\title{
24. COMMISSION DES PARALLAXES STELLAIRES ET DES MOUVEMENTS PROPRES
}

\author{
Report of Meetings, 23 and 25 August 1967
}

President: A. N. Dejč.

SECRETARY: S. Vasilevskis.

In opening the President pointed out that several members of the Commission have not responded to his requests for reports and proposals. Three members have not shown any sign of activity during the last six years, and the President raised the question of possible deletion of these three persons from the list of membership of Commission 24. After some discussions the Commission decided that although the particular persons have retired from astronomical activity, they should be retained as de facto honorary members because of their past contributions.

The following changes in membership were proposed and approved: (1) Delete V. Osvalds on his request. (2) Add A. Blaauw, W. Dieckvoss, N. V. Fatčihin, L. W. Fredrick, A. N. Goyal, W. F. van Altena.

The President announced that on the basis of the membership response to his inquiry, the following officers of the Commission have been nominated for approval by the General Assembly:

President: W. J. Luyten.

Vice-President: S. Vasilevskis.

The Organizing Committee was nominated and approved as follows: N. A. Dejč, V. V. Lavdovskij, J. v. B. Lourens, W.W. Morgan, C.A. Murray, K. Aa. Strand, P. van de Kamp.

The following additions to and changes in the Draft Reports were proposed: Murray of Royal Greenwich Observatory: Symms has determined the parallax and the masses of the components of Polaris by combining visual double star observations with measurements of Greenwich and Allegheny plates.

Meurers of Vienna Observatory: About 2000 proper motions have been determined in the region of $h$ and $\chi$ Per; they are being analyzed for internal motions. The large refractor has been prepared for work on proper motions.

Rodriguez of San Fernando Observatory: 132 plates have been taken of 48 fields with galaxies, on the Pulkovo astrometric program.

Upgren of Van Vleck Observatory: The reference to work on M37 should be changed to A.J. 71, 736, 1966.

The amendments above were approved for inclusion in this report. The President then proposed an agenda for reports and discussions. The agenda was approved and the scientific session proceeded as follows.

Eichhorn reported on reduction of parallax photographs. By using plate constants instead of dependencies one may introduce proper motions of reference stars as unknowns under a condition that the sum of these proper motions is zero. This method yields parallaxes with a higher weight than the conventional procedure of assuming the proper motion of reference stars to be zero.

Lacroute indicated that the method above may be advantageous for large epoch differences but probably there is no advantage if the reference stars have not moved appreciably. Several other members expressed skepticism regarding the merit of the inclusion of proper motions of reference stars as unknown into the plate solution.

Hoffleit reported that photography on the Yale parallax program has been discontinued after leaving the 26-inch refractor in Australia. There are, however, almost 7000 unmeasured photographs in various groups, as stars with only one or no parallax determination, sets of insufficient number of plates for parallax determination, long-series observations of binary stars for orbital motion, and plates taken for proper motions. The present small Yale staff connected with the program is not 
able to measure and reduce the plates in a reasonable time, and the material can be made available to those interested.

In discussions the Commission expressed its concern regarding the Yale program and also regarding the future of the 26-inch refractor. The Commission encourages interested persons to cooperate with Yale.

Fredrick reported that the procedure of parallax determination and the format of publication will be changed at McCormick. He also expressed the need for standard fields of stars for instrumental calibrations. Dieckvoss pointed out that Selected Areas at $+45^{\circ}$ have been suggested earlier $(A . J$. $65,174,1960$ ) for the purpose of calibration.

Upgren reported that the Van Vleck parallax work has been reactivated with an emphasis on faint $M$-dwarfs. Plate constants in $x$ and $y$ will be derived from about 10 reference stars.

Gliese informed that the second revised edition of the Catalogue of Stars within $20 \mathrm{pc}$. will be published. Only approximately 40 stars have been added since 1957 . This number, however, would need a drastic revision upward if the proposals by Schilt and Strand were adopted, i.e. not to reduce the results of various observatories to a common system, in spite of some large systematic differences. In that case all the Yale and Cape parallaxes should be increased by 0".005 and 0".006 respectively. As long as the systematic differences are not explained, there seems to be no reason for preferring a particular observatory. On the other hand, the explanation of these differences is urgent for a reliable system of parallaxes.

Dejč reported that Kiselev at Pulkovo has derived the value of +0 . $075 \pm 0.012$ (m.e.) for the parallax of LP 9-231 which has been called a blue pigmy star. The Pulkovo result indicates that it is a white dwarf.

Morgan reported on the present status of spectroscopic parallaxes. Reliable stellar luminosities can be derived spectroscopically for a limited number of types, not for all the spectral classes. It is not advisable therefore to include spectroscopic parallaxes into a parallax catalogue at present.

After discussions the following resolution was passed:

Commission 24 requests W.W. Morgan to organize and to chair a working group on spectroscopic parallaxes. Members of the working group to be coopted by the Chairman in cooperation with the President of the Commission. It is recommended that the group be formed and a report on spectroscopic parallaxes be prepared before the next General Assembly of the IAU.

Vasilevskis reported that the equipment for automatic measurement of plates has been completed and installed at Lick Observatory by the Gaertner Scientific Corporation. Extensive tests have proved that its performance is in accordance with the original plans $(A . J .65,208,1960)$ and specifications. The equipment consists of the Survey Machine for inspection and selection of objects for measurement, and the Automatic Engine for completely automatic measurement of positions and magnitudes, in a specially conditioned separate room without the presence of an operator. The range of measurement is 17 inches $(430 \mathrm{~mm})$, and the mean error of a measurement over this range is less than one micron; the repeatability is of the order of 0.3 microns.

Strand reported on the new measuring machine for plates up to $10 \times 10 \mathrm{in}$. The positioning, centering and readout is done automatically; each of the three steps is initiated by an operator at the machine. The presence of the operator has no appreciable effect because the main structural components, including the carriages, are made of granite. Air bearings are used for support of moving parts. Centering is performed by a rotating sector with 24 slits 400 microns wide. Ferranty moiré fringe system is used for positioning and readout, the latter to one micron. Images from 70 to 270 microns can be measured, with an accuracy of 0.8 microns. Speed is about 5 times and accuracy about $24 \%$ higher than with the conventional machines.

Luyten discussed briefly his new derivation of the Luminosity function based on proper motion data obtained from a survey made on 117 plates of the Palomar Schmidt Survey. More than 50000 moving objects were found brighter than $21.5 \mathrm{pg}$ and with motions larger than 0 ". 04 annually. Using solar apex determinations to calculate mean parallaxes a luminosity function was derived which is found to have a maximum at $M=+15.7 \mathrm{pg}$. The total number of stars nearer than 10 parsecs was found to be between 550 and 600 and the total mass of luminous objects at $0.064 \odot$ per cubic parsec. 
Fatčihin reported on preliminary results from stellar proper motions determined with reference to galaxies. The absolute motions of 14000 stars, including 1283 AGK3 stars were related to 271 galaxies in 82 areas photographed with the Pulkovo normal astrograph, the average epoch difference being 22.4 years. The values of $A=302: 5 \pm 4: 5, D=32: 6 \pm 2: 7$ were found for the solar apex from stars of approximate photographic magnitude 15 . The mean secular parallax of these stars increases with the galactic latitude, from 0 ".0047 to 0 ".00112, with the probable errors of the order of \pm 0 ". 001 for each of three zones investigated. A comparison with the AGK3 yielded the correction to the Newcomb precession: $\Delta m=-0.0053 \pm 0 " .0011$ and $\Delta n=0 " .0044 \pm 0 " 0011$. Systematic differences with the AGK3 were also studied.

Dieckvoss reported that AGK3 proper motions of 163811 stars were statistically investigated for solar motion, secular parallax, galactic rotation and dispersion. The solutions gave the following values for correction to centennial precession and for Oort's constants in $\mathrm{km} / \mathrm{sec} / \mathrm{kpc}$, with their mean errors: $\Delta m=-0$ ". $32 \pm 0$ ".01, $\Delta n=+0$ ". $51 \pm 0$ ".01, $A=+14.8 \pm 0.7, B=11.3 \pm 0.6$.

Giclas reported on the Lowell proper motion program. More than 12000 stars with annual motions larger than 0 ". 26 have been listed in 12 Lowell Bulletins, and an additional number of approximately 5000 stars is expected to be published in the same way. A question was raised whether eventually a catalog of all the stars arranged in RA should be published.

After discussions Commission 24 recommended:

It would be very useful to publish a catalogue of Lowell Survey stars, after the survey is completed. All the duplicate measurements should be combined, and the stars listed in the sequence of their right ascension, with references to the original finding charts.

Dejč reported on the progress of the Pulkovo program of proper motions with reference to galaxies in the zone $-25^{\circ}$ to $-90^{\circ}$. The Observatories at Santiago, Perth, Cape and Cordoba are participating with their normal astrographs; it is hoped that La Plata will also join. In addition, a new Maksutov type telescope has been installed $100 \mathrm{~km}$ north of Santiago, with the focal length, meniscus diameter and field of $2 \mathrm{~m}, 70 \mathrm{~cm}$ and $5^{\circ}$ respectively. A list of 153 fundamental stars of FKSZ in the same zone has been prepared for observing with normal astrographs, or preferably with longer telescopes of focal length 5 to $6 \mathrm{~m}$.

After discussions Commission 24 concluded:

That it is important to observe urgently the 153 fundamental stars with telescopes mentioned above. The Commission recommends that southern observatories having such telescopes participate in the program. 